\title{
ON WELL-BOUNDED OPERATORS OF TYPE (B)
}

\author{
by P. G. SPAIN \\ (Received 3rd June 1971)
}

\section{Introduction}

The notion of a well-bounded operator was introduced by Smart (9). The properties of well-bounded operators were further investigated by Ringrose $(6,7)$, Sills (8) and Berkson and Dowson (2). Berkson and Dowson have developed a more complete theory for the type (A) and type (B) well-bounded operators than is possible for the general well-bounded operator. Their work relies heavily on Sills' treatment of the Banach algebra structure of the second dual of the Banach algebra of absolutely continuous functions on a compact interval.

The main result of this paper (Theorem 5) is the characterisation of a type (B) operator by means of the weak compactness of its $\mathscr{A}_{\mathrm{J}}$-operational calculus (as in Theorem 4.2 of (2)) and the description of the operational calculus using Stieltjes integrals of a kind suggested by Krabbe (5). Our results are also stronger than those of Berkson and Dowson in that we show the $\mathscr{B}_{J}$-operational calculus for a type (B) operator to be continuous on pointwise convergent nets of uniformly bounded variation.

We are indebted to $H$. R. Dowson for much valuable advice and encouragement during the preparation of this paper.

\section{Preliminaries}

Let $X$ be a complex Banach space with dual space $X^{\prime}$. We write $\left\langle x, x^{\prime}\right\rangle$ for the value of the functional $x^{\prime}$ in $X^{\prime}$ at $x$ in $X$. When $Y$ is a subset of $X$ we write $Y^{w}$ for the weak closure of $Y$, and $Y_{1}$ for $\{y \in Y:\|y\| \leqq 1\}$.

Let $L(X)$ be the Banach algebra of bounded linear operators on $X$. When $T$ is in $L(X)$, let $T^{\prime}$ in $L\left(X^{\prime}\right)$ be its adjoint.

We shall abbreviate "weak/strong operator topology" to "weak/strong topology". When $\mathscr{T}$ is a subset of $L(X)$, we write $\mathscr{T}^{w}$ and $\mathscr{T}^{s}$ for the weak and strong closures of $\mathscr{T}$. We write "wk lim" and "st lim" for limits in the weak and strong topologies.

Lemma 1. $\mathscr{T}$ is relatively weakly compact in $L(X)$ if and only if $\mathscr{T} x$ is relatively weakly compact in $X$ (for every $x$ in $X$ ).

Proof. The proof is as indicated in (3, VI, 9.2).

Let $\left\{T_{\alpha}: \alpha \in \sigma\right\}$ be a net in $L(X)$. Following (1) we say that $y$ is a weak $x$-cluster point of $\left\{T_{\alpha}\right\}$ if $y$ is a weak cluster point of the net $\left\{T_{\alpha} x: \alpha \in \sigma\right\}$. 
An operator $E$ in $L(X)$ is called a projection if $E^{2}=E$. We write $E \leqq F$ when $E$ and $F$ are two projections such that $E F=F E=E$. When $E$ and $F$ are commuting projections both $E \vee F(=E+F-E F)$ and $E \wedge F(=E F)$ are also projections.

A net $\left\{E_{\alpha}: \alpha \in \sigma\right\}$ of projections is said to be naturally ordered if $E_{\alpha} \leqq E_{\beta}$ whenever $\alpha \leqq \beta$.

Lemma 2. Let $\left\{E_{\alpha}: \alpha \in \sigma\right\}$ be a naturally ordered uniformly bounded net of projections on $X$. Then $\bigvee_{\sigma} E_{\alpha}$ exists, and is equal to st $\lim _{\sigma} E_{\alpha}$, if and only if $\left\{E_{\alpha}\right\}$ has a weak $x$-cluster point for each $x$ in $X$.

Proof. (1, Theorem 1.)

We shall extend this terminology and say that the net $\left\{E_{\alpha}: \alpha \in \sigma\right\}$ is a naturally ordered net of operators if $E_{\alpha}=E_{\alpha} E_{\beta}=E_{\beta} E_{\alpha}$ whenever $\alpha<\beta$; we do not require the operators $E_{\alpha}$ to be projections.

Lemma 3. Let $\left\{E_{\alpha}: \alpha \in \sigma\right\}$ be a naturally ordered uniformly bounded net of operators on $X$. Then st $\lim E_{\alpha}$ exists if and only if $\left\{E_{\alpha}\right\}$ has a weak $x$-cluster point for each $x$ in $X$.

Proof. The proof is a straightforward adaptation of the proof of (1, Theorem 1) and is therefore omitted.

We say that $E: R \rightarrow L(X)$ is a naturally ordered function if

$$
E(s)=E(s) E(t)=E(t) E(s)
$$

for $s<t$. We write $E(s+)$ for st $\lim E(t)$ and $E(s-)$ for st $\lim E(t)$ when the limits exist.

Let $T$ be an operator on $X$. By an $(\mathscr{F}$-)operational calculus for $T$ we mean a bounded algebra homomorphism $\psi: \mathscr{F} \rightarrow L(X)$, where $\mathscr{F}$ is a normed algebra of functions on a compact subset of the complex plane $C, \mathscr{F}$ contains the functions $\lambda \mapsto 1, \lambda \mapsto \lambda$, and $\psi(\lambda \mapsto 1)=I, \psi(\lambda \mapsto \lambda)=T$. We write $\psi(f)$ and $f(T)$ interchangeably. For each $x$ in $X$ and $x^{\prime}$ in $X^{\prime}$ we define $\psi_{x}: \mathscr{F} \rightarrow X: f \mapsto \psi(f) x$ and $\psi_{x, x^{\prime}}: \mathscr{F} \rightarrow C: f \mapsto\left\langle\psi(f) x, x^{\prime}\right\rangle$.

Let $J=[a, b]$ be a compact interval in the real line $R$. Let $\mathscr{B}_{J}$ be the Banach algebra of complex-valued functions of bounded variation on $J$ with norm \|\|$\cdot\|\|_{J},\|f\|_{J}=|f(b)|+\operatorname{var}(f, J)$. Let $\mathscr{A}_{J}$ be the Banach subalgebra of absolutely continuous functions on $J$. For $f$ in $\mathscr{A}_{J}$,

$$
\|f\|_{J}=|f(b)|+\int_{a}^{b}\left|f^{\prime}(\lambda)\right| d \lambda .
$$

Let $\mathscr{A}_{J}^{0}$ and $\mathscr{B}_{J}^{0}$ be the Banach subalgebras

$$
\left\{f \in \mathscr{A}_{J}: f(b)=0\right\} \text { and }\left\{f \in \mathscr{B}_{J}: f(b)=0\right\}
$$


of $\mathscr{A}_{J}$ and $\mathscr{B}_{J}$. Let $\mathscr{N}_{J}$ be the Banach subalgebra of $\mathscr{B}_{J}$ consisting of the functions in $\mathscr{B}_{J}$ which are left continuous on $(a, b]$. Let $\mathscr{P}_{J}$ be the subalgebra of $\mathscr{A}_{J}$ consisting of the polynomials on $J . \mathscr{P}_{J}$ is dense in $\mathscr{A}_{J}$.

\section{Integration theory}

The integrals described here are based on the modified Stieltjes integrals of Krabbe (5).

Let $\mathscr{E}_{J}(J=[a, b])$ be the family of functions $E: R \rightarrow L(X)$ satisfying

(i) $E(s-)$ exists, $\quad s \in R$;

(ii) $E(s)=E(s+), \quad s \in R$;

(iii) $E(s)=0, \quad \cdot s<a$;

(iv) $E(s)=E(b), \quad s \geqq b$.

It is clear that $\sup _{R}\|E(s)\|=\sup _{J}\|E(s)\|<\infty$ for $E$ in $\mathscr{E}_{J}$.

We say that a sequence $u=\left(u_{k}: 0 \leqq k \leqq m\right)$ is a subdivision of $J$ if $a=u_{0}<u_{1}<\ldots<u_{m}=b$. The set $U_{J}$ of all subdivisions of $J$ admits a partial order $\geqq$ defined by refinement: we write

$$
u=\left(u_{k}: 0 \leqq k \leqq m\right) \geqq v=\left(v_{j}: 0 \leqq j \leqq n\right)
$$

when $u$ refines $v$; that is, when each $\left[u_{k-1}, u_{k}\right](1 \leqq k \leqq m)$ is contained in some $\left[v_{j-1}, v_{j}\right](1 \leqq j \leqq n)$.

Let $M(u)$ be the family of sequences $u^{*}=\left(u_{k}^{*}: 1 \leqq k \leqq m\right)$ such that

$$
u_{k-1} \leqq u_{k}^{*} \leqq u_{k}(1 \leqq k \leqq m),
$$

for each $u$ in $U_{J}$.

A pair $\bar{u}=\left(u, u^{*}\right)$ with $u \in U_{J}$ and $u^{*} \in M(u)$ is called a marked partition of $J$. We write $\pi_{J}$ for the family of marked partitions of $J$ and define the pre-order $\geqq$ on $\pi_{J}$ by setting $\left(u, u^{*}\right) \geqq\left(v, v^{*}\right)$ if and only if $u \geqq v$.

Let $\pi_{J}^{i}=\left\{\bar{u}=\left(u, u^{*}\right) \in \pi_{J}: u_{k-1}<u_{k}^{*}<u_{k}, 1 \leqq k \leqq m\right\}$ and let

$$
\pi_{J}^{r}=\left\{\bar{u}=\left(u, u^{*}\right) \in \pi_{J}: u_{k}^{*}=u_{k}, 1 \leqq k \leqq m\right\} .
$$
in $\pi_{J}$.

The sets $U_{J}, \pi_{J}, \pi_{J}^{i}$ and $\pi_{J}^{r}$ are directed by $\geqq$. Also, $\pi_{J}^{i}$ and $\pi_{J}^{r}$ are cofinal

We define $\pi_{J}^{g}$ for each $g$ in $\mathscr{B}_{J}$ thus:

$$
\pi_{J}^{g}= \begin{cases}\pi_{J}, & g \in \mathscr{N}_{J}, \\ \pi_{J}^{i}, & g \in \mathscr{B} \backslash \mathcal{N}_{J} .\end{cases}
$$

Let $E_{u}=\sum_{1}^{m} E\left(u_{k-1}\right) \chi_{\left[u_{k-1}, u_{k}\right)}+E(b) \chi_{[b, \infty)}$ when $E \in \mathscr{E}_{J}$ and $u \in U_{J}$.

Let $g_{\bar{u}}=g(a) \chi_{\{a\}}+\sum_{1}^{m} g\left(u_{k}^{*}\right) \chi_{\left(u_{k-1}, u_{k}\right]}$ when $g \in \mathscr{B}_{J}$ and $\bar{u} \in \pi_{J}$. 
Let $\Phi$ and $\Psi$ be functions on $J$, one taking values in $C$, the other in $L(X)$ or in $C$. When $\bar{u} \in \pi_{J}$ we define

$$
\Sigma \Phi(\Psi \Delta \bar{u})=\sum_{1}^{m} \Phi\left(u_{k}^{*}\right)\left(\Psi\left(u_{k}\right)-\Psi\left(u_{k-1}\right)\right)
$$

The following two inequalities are evident:

$$
\begin{aligned}
& \|\Sigma E(g \Delta \bar{u})-\Sigma F(g \Delta \bar{u})\| \leqq \operatorname{var}(g, J) \sup _{J}\|E(s)-F(s)\|, g \in \mathscr{B}_{J}, E, F \in \mathscr{E}_{J} ; \\
& \|\Sigma E(g \Delta \bar{u}) x-\Sigma F(g \Delta \bar{u}) x\| \leqq \operatorname{var}(g, J) \sup _{J}\|E(s) x-F(s) x\|, \\
& x \in X, g \in \mathscr{B}_{J}, E, F \in \mathscr{E}_{J} .
\end{aligned}
$$

The following integrals are defined as net limits in the strong topology (when they exist):

$$
\begin{aligned}
& \int_{J} \Phi d \Psi=\mathrm{st} \lim \Sigma \Phi(\Psi \Delta \bar{u}) \\
& \int_{\pi_{J}}^{r} \Phi d \Psi=\mathrm{st} \lim _{\pi_{J}^{r}} \Sigma \Phi(\Psi \Delta \bar{u}) ; \\
& \int_{J}^{i} \Phi d \Psi=\text { st } \lim _{\pi_{J}^{\prime}} \Sigma \Phi(\Psi \Delta \bar{u}) ; \\
& \oint_{J} E d g=\mathrm{st} \lim _{\pi_{J}^{g}} \Sigma E(g \Delta \bar{u}), \quad g \in \mathscr{B}_{J}, E \in \mathscr{E}_{J} .
\end{aligned}
$$

Lemma 4. $\lim _{U_{J}} \sup _{J}\left\|E(s) x-E_{u}(s) x\right\|=0, x \in X, E \in \mathscr{E}_{J}$.

Proof. Let $E \in \mathscr{E}_{J}, x \in X$. Let $\varepsilon>0$.

For each $s$ in $[a, b)$ there exists $r_{s}\left(s<r_{s}<b\right)$ such that $\left\|E(t) x-E\left(t^{\prime}\right) x\right\| \leqq \varepsilon$ when $t, t^{\prime} \in\left[s, r_{s}\right)$, since $E(s)=E(s+)$.

For each $s$ in $(a, b]$ there exists $l_{s}\left(a<l_{s}<s\right)$ such that $\left\|E(t) x-E\left(t^{\prime}\right) x\right\| \leqq \varepsilon$ when $t, t^{\prime} \in\left[l_{s}, s\right)$, since $E(s-)$ exists.

The sets $\left[a, r_{a}\right),\left(l_{b}, b\right],\left(l_{s}, r_{s}\right)(a<s<b)$ form an open cover of $J$. Let $\left[a, r_{a}\right),\left(l_{b}, b\right],\left(l_{s}, r_{s_{j}}\right)(j$ in some finite set) be a finite subcover.

Let $u$ be the partition of $J$ with points $a, b, r_{a}, l_{b}, s_{j}, l_{s_{j}}, r_{s_{j}}(j$ in the finite set). Then $\sup _{J}\left\|E(s) x-E_{u}(s) x\right\| \leqq \varepsilon$.

\section{Lemma 5.}
(i) $\oint_{J} T \chi_{[b, \infty)} d g=0, \quad g \in \mathscr{B}_{J}, T \in L(X)$;
(ii) $\oint_{J} T \chi_{[s, t)} d g=(g(t)-g(s)) T, \quad g \in \mathscr{B}_{J}, T \in L(X), a \leqq s<t \leqq b$; 
(iii) $\oint_{J} E_{u} d g=\sum_{1}^{m} E\left(u_{k-1}\right)\left(g\left(u_{k}\right)-g\left(u_{k-1}\right)\right)$

$$
=s t \lim _{\pi_{J}^{s}} \Sigma E_{u}(g \Delta \bar{v}), \quad g \in \mathscr{B}_{J}, E \in \mathscr{E}_{J}, u \in U_{J} .
$$

Proof. (i) Let $\bar{u} \in \pi_{J}^{g}$. Then

$$
\begin{aligned}
\Sigma \chi_{[b, \infty)}(g \Delta \bar{u}) & =\sum_{1}^{m} \chi_{[b, \infty)}\left(u_{k}^{*}\right)\left(g\left(u_{k}\right)-g\left(u_{k-1}\right)\right) \\
& = \begin{cases}g(b)-g\left(u_{m-1}\right), & u_{m}^{*}=b, \\
0, & u_{m}^{*}<b .\end{cases}
\end{aligned}
$$

Hence st $\lim \Sigma T \chi_{[b, \infty)}(g \Delta \bar{u})=0$.

(ii) Let $a<s \leqq b, \bar{u} \in \pi_{J}^{g}, u \geqq(a, s, b)$ (no condition if $s=b$ ). Then $s=u_{n}$ for some $n$ with $1 \leqq n \leqq m$, and

$$
\begin{aligned}
\Sigma \chi_{[a, s)}(g \Delta \bar{u}) & =\sum_{1}^{n-1}\left(g\left(u_{k}\right)-g\left(u_{k-1}\right)\right)+\chi_{[a, s)}\left(u_{n}^{*}\right)\left(g(s)-g\left(u_{n-1}\right)\right) \\
& = \begin{cases}g\left(u_{n-1}\right)-g(a), & u_{n}^{*}=u_{n}=s, \\
g(s)-g(a), & u_{n}^{*}<u_{n}=s .\end{cases}
\end{aligned}
$$

Hence st $\lim \Sigma T \chi_{[a, s)}(g \Delta \bar{u})=(g(s)-g(a)) T$. Since $\pi g_{j}$

(ii) is proved.

$$
\chi_{[s, t)}=\chi_{[a, t)}-\chi_{[a, s)}(a \leqq s<t \leqq b),
$$

(iii) is a direct corollary of (i) and (ii).

Theorem 1. Let $g$ be in $\mathscr{B}_{J}$ and $E$ in $\mathscr{E}_{J}$. Then $\oint_{J}$ Edg exists, and

$$
\oint_{J} E d g=\text { st } \lim _{U_{J}} \oint_{J} E_{u} d g
$$

Also,

$$
\begin{gathered}
\left\|\oint_{J} E d g\right\| \leqq \operatorname{var}(g, J) \sup _{J}\|E(s)\|, \\
\left\|\oint_{J} E d g x\right\| \leqq \operatorname{var}(g, J) \sup _{J}\|E(s) x\|, \quad x \in X .
\end{gathered}
$$

Proof. By (1) above,

$$
\|\Sigma E(g \Delta \bar{v})\| \leqq \operatorname{var}(g, J) \sup _{J}\|E(s)\|, \quad \bar{v} \in \pi_{J}
$$


Let $u \in U_{J}$, let $\bar{v}, \bar{w} \in \pi_{J}^{g}$ and let $x \in X$. Then

$$
\begin{aligned}
\| \Sigma E(g \Delta \bar{v}) x- & \Sigma E(g \Delta \bar{w}) x\|\leqq\| \Sigma E(g \Delta \bar{v}) x-\Sigma E_{u}(g \Delta \bar{v}) x \| \\
& +\left\|\Sigma E(g \Delta \bar{w}) x-\Sigma E_{u}(g \Delta \bar{w}) x\right\|+\left\|\Sigma E_{u}(g \Delta \bar{v}) x-\Sigma E_{u}(g \Delta \bar{w}) x\right\| \\
& \leqq 2 \operatorname{var}(g, J) \sup _{J}\left\|E(s) x-E_{u}(s) x\right\|+\left\|\Sigma E_{u}(g \Delta \bar{v}) x-\Sigma E_{u}(g \Delta \bar{w}) x\right\| .
\end{aligned}
$$

Lemmas 4 and 5 now show that $\|\Sigma E(g \Delta \bar{v}) x-\Sigma E(g \Delta \bar{w}) x\| \rightarrow 0$ as $\bar{v}$ and $\bar{w}$ increase in $\pi_{J}^{g}$. Therefore $\left\{\Sigma E(g \Delta \bar{v}) ; \bar{v} \in \pi_{J}^{g}\right\}$ is a uniformly bounded strongly Cauchy net in $L(X)$, and so converges to its unique strong limit.

The other statements of the theorem are immediate from (1) and (2).

Theorem 2. Let $E \in \mathscr{E}_{J}, g \in \mathscr{B}_{J}$ and let $\left\{g_{\alpha}: \alpha \in \sigma\right\}$ be a net in $\mathscr{B}_{J}$ with $\sup _{\sigma} \operatorname{var}\left(g_{\alpha}, J\right)<\infty$ and $g(s)=\lim _{\sigma} g_{\alpha}(s)(s \in J)$. Then

$$
\oint_{J} E d g=\operatorname{st} \lim _{\sigma} \oint_{J} E d g_{\alpha}
$$

Proof. Let $K=\operatorname{var}(g, J)+\sup _{\sigma} \operatorname{var}\left(g_{\alpha}, J\right)$. Let $u \in U_{J}$. Then

$$
\oint_{J} E d g-\oint_{J} E d g_{\alpha}=\oint_{J}\left(E-E_{u}\right) d g-\oint_{J}\left(E-E_{u}\right) d g_{\alpha}+\oint_{J} E_{u} d\left(g-g_{\alpha}\right) .
$$

Let $x \in X$. Then

$$
\begin{aligned}
\left\|\oint_{J} E d g x-\oint_{J} E d g_{\alpha} x\right\| & \leqq K \sup _{J}\left\|E(s) x-E_{u}(s) x\right\| \\
& +\sup _{J}\|E(s) x\| \sum_{1}^{m}\left|\left(g-g_{\alpha}\right)\left(u_{k}\right)-\left(g-g_{\alpha}\right)\left(u_{k-1}\right)\right|,
\end{aligned}
$$

and this expression can be made arbitrarily small by choosing $u$ fine enough (Lemma 4), then $\alpha$ large enough.

Let $S(g, E)=g(b) E(b)-\oint_{J} E d g$ when $g \in \mathscr{B}_{J}, \quad E \in \mathscr{E}_{\mathrm{J}}$.

\section{Lemma 6.}

(i) $S\left(g, \chi_{[s, \infty)} T\right)=g(s) T, \quad g \in \mathscr{B}_{J}, T \in L(X), a \leqq s \leqq b ;$

(ii) $\|S(g, E)\| \leqq\|\mid g\|_{J} \sup _{J}\|E(s)\|, \quad g \in \mathscr{B}_{J}, E \in \mathscr{E}_{J}$;

(iii) $\|S(g, E) x\| \leqq\|g\|_{J} \sup _{J}\|E(s) x\|, \quad g \in \mathscr{B}_{J}, E \in \mathscr{E}_{J}, x \in X$;

(iv) $S\left(\chi_{[a, s]}, E\right)=E(s), \quad E \in \mathscr{E}_{J}, s \in J$.

Proof. (i), (ii) and (iii) follow directly from Lemma 5 and Theorem 1. As to (iv):

(a) $s=b . \quad S\left(\chi_{J}, E\right)=1 \cdot E(b)-\oint_{J} E d \chi_{J}=E(b)$ 
(b) $s<b$. Then

$$
\begin{aligned}
S\left(\chi_{[a, s]}, E\right) & =-\oint_{J} E d \chi_{[a, s]} \\
& =-s \lim _{U_{J}} \oint_{J} E_{u} d \chi_{[a, s]} \quad \text { (Theorem 1) } \\
& =-s t \lim _{U_{J}} \sum_{1}^{m} E\left(u_{k-1}\right)\left(\chi_{[a, s]}\left(u_{k}\right)-\chi_{[a, s]}\left(u_{k-1}\right)\right) \\
& =-s t \lim _{U_{J}}\left(-E\left(u_{n-1}\right)\right) \text { where } s \in\left[u_{n-1}, u_{n}\right) \\
& =\text { st } \lim _{U J} E_{u}(s) \\
& =E(s) \quad \text { (Lemma 4). }
\end{aligned}
$$

Lemma 7. Let $g \in \mathscr{B}_{J}$ and $\bar{u} \in \pi_{J}^{g}$. Then $g_{\bar{u}} \in \mathscr{B}_{J}$, and

$$
g(s)=\lim _{\pi_{j}} g_{\bar{u}}(s), \quad s \in J .
$$

Also, $\operatorname{var}\left(g_{\vec{u}}, J\right) \leqq 2 \sup _{J}|g(s)|$ if $g$ is real monotonic increasing.

Proof. $g_{\bar{u}} \in \mathscr{B}_{J}$, trivially; and $g_{\bar{u}}(a)=g(a)$. If $a<s \leqq b$ and $u \geqq(a, s, b)$ (no condition if $s=b$ ) then $s=u_{n}$ for some $n(1 \leqq n \leqq m)$ and

$$
g_{\bar{u}}(s)=g\left(u_{n}^{*}\right)= \begin{cases}g\left(u_{n}^{*}\right), & g \in \mathscr{N}_{J}, \\ g\left(u_{n}\right), & g \in \mathscr{B}_{J} \backslash \mathscr{N}_{J} .\end{cases}
$$

Therefore $\lim _{\pi^{g}} g_{\bar{u}}(s)=g(s)(s \in J)$.

If $g$ is real monotonic increasing, then $\operatorname{var}(g, J) \leqq 2$ sup $|g(s)|$ and $g_{\bar{u}}$ is also monotonic increasing. Hence $\operatorname{var}\left(g_{\bar{u}}, J\right) \leqq 2 \sup _{J}\left|g_{\bar{u}}(s)\right| \leqq 2 \sup _{J}|g(s)|$.

Theorem 3. Let $E \in \mathscr{E}_{J}$. Then

$$
S(g, E)= \begin{cases}g(a) E(a)+\int_{J} g d E, & g \in \mathscr{N}_{J}, \\ g(a) E(a)+\int_{J}^{r} g d E, & g \in \mathscr{B}_{\mathrm{J}} .\end{cases}
$$

Proof. Let $\bar{u} \in \pi_{J}^{g}$. Then

$$
\begin{aligned}
S\left(g_{\bar{u}}, E\right) & =g(a) S\left(\chi_{\{a\}}, E\right)+\sum_{1}^{m} g\left(u_{k}^{*}\right)\left(S\left(\chi_{\left[a, u_{k}\right]}, E\right)-S\left(\chi_{\left[a, u_{k}-1\right]}, E\right)\right) \\
& =g(a) E(a)+\Sigma g(E \Delta \bar{u}) .
\end{aligned}
$$


We can assume that $g$ is real monotonic increasing, without loss of generality. Theorem 2 and Lemma 7 now give the result.

We shall write $\int_{J}^{\oplus} g d E$ instead of $S(g, E)$ when $g \in \mathscr{B}_{J}$ and $E \in \mathscr{E}_{J}$.

The proofs above give us analogous scalar integrals defined for functions of bounded variation on $J$ and functions satisfying the scalar version of the definition of $\mathscr{E}_{J}$.

Let $\mathscr{D}_{J}$ be the algebra of complex-valued functions on $R$ generated by the functions $\chi_{[s, t)}(a \leqq s<t \leqq b)$. Let $\mathscr{Q}_{J}$ be the closure of $\mathscr{D}_{J}$ in the supremum norm. Then $\mathscr{Q}_{J}$ is the algebra of functions which vanish on $(-\infty, a)$ and on $[b, \infty)$ and are right continuous and left limitable on $\boldsymbol{R}$ (Lemma 4 or (4, Theorem 4.5)).

Hewitt showed in (4) that the integral $\int_{[a, b)} \omega d g$ can be defined for $g$ in $\mathscr{B}_{J}$ and $\omega$ in $\mathscr{Q}_{J}$ as the limit of any sequence $\left\{\int_{[a, b)} \omega_{n} d g\right\}$ where $\left\{\omega_{n}\right\} \subset \mathscr{D}_{J}$, $\omega=\lim \omega_{n}$ in the supremum norm, and $\int_{[a, b)} \chi_{[s, t)} d g=g(t)-g(s)(a \leqq s<t \leqq b)$ by definition. The scalar version of Theorem 1 shows that

$$
\int_{[a, b)} \omega d g=\int_{J}^{i} \omega d g \quad\left(g \in \mathscr{B}_{J}, \omega \in \mathscr{Q}_{J}\right) .
$$

Let $\Sigma_{J}$ be the algebra of subsets of $[a, b)$ generated by sets of the form $[s, t)(a \leqq s<t \leqq b)$. Theorem 4.10 of (4) shows that $\left(\mathscr{Q}_{J}\right)^{\prime}$ may be identified with the space of bounded finitely additive measures on $\Sigma_{\boldsymbol{J}}$. Each such measure $\mu$ defines a function $g_{\mu}$ in $\mathscr{B}_{J}^{0}$ by $g_{\mu}(s)=-\mu([s, b))(s \in J)$ : conversely, each function $g$ in $\mathscr{B}_{J}^{0}$ defines such a measure $\mu_{g}$ by $\mu_{\theta}([s, t))=g(t)-g(s)(a \leqq s<t \leqq b)$. This correspondence is one-to-one.

We can therefore identify $\left(\mathscr{Q}_{J}\right)^{\prime}$ and $\mathscr{B}_{\mathrm{J}}^{0}$. The pairing between $\mathscr{Q}_{J}$ and $\mathscr{B}_{J}^{0}$ is given by

$$
\begin{aligned}
\langle\omega, g\rangle & =\int_{[a, b)} \omega d g, \\
& =\int_{J}^{i} \omega d g, \quad \omega \in \mathscr{Q}_{J}, g \in \mathscr{B}_{J}^{0} .
\end{aligned}
$$

Lemma 8. Let $\left\{g_{a}: \alpha \in \sigma\right\}$ be a bounded net in $\mathscr{B}_{J}^{0}$ and let $g \in \mathscr{B}_{J}^{0}$. Then $g=\lim _{\sigma} g_{\alpha}$ in the $\mathscr{Q}_{J}$-topology of $\mathscr{B}_{J}^{0}$ if and only if $g(s)=\lim _{\sigma} g_{\alpha}(s)(a \leqq s<b)$.

Proof. (Cf. (3, IV, 13.35).) Note that $\left\langle\chi_{[s, b)}, g\right\rangle=-g(s)(a \leqq s<b)$ and apply Theorem 2 .

The following result seems not to be known generally. 
Theorem 4. Let $g \in \mathscr{B}_{J}$. Then there is a net $\left\{g_{\alpha}: \alpha \in \sigma\right\}$ in $\mathscr{A}_{J}$ such that $g=\lim _{\sigma} g_{\alpha}$ pointwise on $J$ and $\sup \left\|g_{\alpha}\right\|_{J} \leqq\|g\|_{J}$.

Proof. Since $g$ can be written as $\left(g-g(b) \chi_{J}\right)+g(b) \chi_{J}$ we see that it is enough to show that if $g \in \mathscr{B}_{J}^{0}$ then there is a net $\left\{g_{\alpha}: \alpha \in \sigma\right\}$ in $\mathscr{A}_{J}^{0}$ such that $g=\lim _{\sigma} g_{\alpha}$ pointwise on $[a, b)$ and $\sup \operatorname{var}\left(g_{a}, J\right) \leqq \operatorname{var}(g, J)$.

Now each $\omega$ in $\mathscr{Q}_{J}$ defines a bounded functional on $\mathscr{A}_{J}^{0}$ by $f \mapsto \int_{J} \omega d f$. Therefore $\mathscr{Q}_{J}$ can be identified with a subspace of $\left(\mathscr{A}_{J}^{0}\right)^{\prime}$, that is, with $L^{\infty}(J)$ under the ess-sup norm.

Each function $g$ in $\mathscr{B}_{J}^{0}$ defines a bounded functional $L_{g}$ on $\mathscr{Q}_{J}$ by

$$
L_{g}(\omega)=\int_{J}^{i} \omega d g \quad\left(\omega \in \mathscr{Q}_{J}\right)
$$

Since the sup and ess-sup norms agree on $\mathscr{Q}_{J}$, we see from Theorem 1 that

$$
\left\|L_{g}\right\| \leqq\|g\|_{J}=\operatorname{var}(g, J)
$$

The Hahn-Banach theorem allows us to extend $L_{g}$ to a functional (also denoted by) $L_{g}$ on $\left(\mathscr{A}_{J}^{0}\right)^{\prime}$ without increasing its norm. So $L_{g} \in\left(\mathscr{A}_{J}^{0}\right)^{\prime \prime}$. By Goldstine's theorem $(3, \mathrm{~V}, 4.5)$ there is a net $\left\{g_{\alpha}: \alpha \in \sigma\right\}$ in $\mathscr{A}_{J}^{0}$ converging to $L_{g}$ in the $\left(\mathscr{A}_{J}^{0}\right)^{\prime}$-topology of $\left(\mathscr{A}_{J}^{0}\right)^{\prime \prime}$ and satisfying sup $\left\|g_{\alpha}\right\|_{J} \leqq\left\|L_{g}\right\| \leqq\|g\|_{J}$. Then $g=\lim _{\sigma} g_{\alpha}$ in the $\mathscr{Q}_{J}$-topology of $\mathscr{B}_{J}^{0}$, so $g(s)=\lim _{\sigma} g_{\alpha}(s)(a \leqq s<b)$ (Lemma 8).

\section{Well-bounded operators of type (B)}

Let $T$ be a bounded operator on the Banach space $X$. We define $p(T)$ in the natural way for each polynomial $p$ by setting $p(T)=\Sigma a_{n} T^{n}$ when $p(s)=\Sigma a_{n} s^{n}$. The map $p \mapsto p(T)$ is an algebra homomorphism.

We say that $T$ is well-bounded (on $J$ ) if there is a compact interval $J$ such that $\psi: \mathscr{P}_{\mathrm{J}} \rightarrow L(X): p \mapsto p(T)$ is an operational calculus; that is, $T$ is wellbounded if there exist a compact interval $J$ and a constant $K$ such that

$$
\|p(T)\| \leqq K\|p\| J, \quad p \in \mathscr{P}_{J} .
$$

If $T$ is well-bounded, so is $T^{\prime}$ (with the same $J$ and $K$ ).

Smart introduced this definition and proved the following result.

Lemma 9. Let $T$ in $L(X)$ be a well-bounded operator with natural operational calculus $\psi: \mathscr{P}_{J} \rightarrow L(X)$ of norm $K$. Then $\psi$ has a unique extension to an operational calculus (also denoted by) $\psi: \mathscr{A}_{\mathrm{J}} \rightarrow L(X)$, of norm $K$, such that

(i) if $S$ in $L(X)$ satisfies $T S=S T$, then $S f(T)=f(T) S, f \in \mathscr{A}_{J}$;

(ii) $f\left(T^{\prime}\right)=f(T)^{\prime}, f \in \mathscr{A}_{J}$.

Proof. (9, Lemma 2.1.) 
The notion of a decomposition of the identity was introduced by Ringrose in (7). A decomposition of the identity for $X$ (on $J$ ) is a family $\{F(s): s \in R\}$ of projections on $X^{\prime}$ such that

(i) $F(s)=0, s<a$,

$$
F(s)=I, \quad s \geqq b ;
$$

(ii) $F(s) F(t)=F(t) F(s)=F(s), \quad s \leqq t$;

(iii) there is a positive constant $K(\geqq 1)$ such that

$$
\|F(s)\| \leqq K, \quad s \in R
$$

(iv) the function $s \mapsto\left\langle x, F(s) x^{\prime}\right\rangle$ is Lebesgue measurable for each $x \in X$ and $x^{\prime} \in X^{\prime}$;

(v) if $x \in X, x^{\prime} \in X^{\prime}, s \in[a, b)$, and if the function $t \mapsto \int_{a}^{t}\left\langle x, F(u) x^{\prime}\right\rangle d u$ is right differentiable at $s$, then the right derivative at $s$ is $\left\langle x, F(s) x^{\prime}\right\rangle$;

(vi) for each $x$ in $X$, the map $X^{\prime} \rightarrow L^{\infty}(a, b): x^{\prime} \mapsto\langle x, F(\quad) x\rangle$ is continuous when $X^{\prime}$ and $L^{\infty}(a, b)$ are) given their weak* topologies (as duals of $X$ and $\left.L^{1}(a, b)\right)$.

An operator $T$ in $L(X)$ is said to be decomposable (on $J$ ) if there is a decomposition of the identity for $X$ on $J$ such that

$$
\left\langle T x, x^{\prime}\right\rangle=b\left\langle x, x^{\prime}\right\rangle-\int_{a}^{b}\left\langle x, F(s) x^{\prime}\right\rangle d s, \quad x \in X, x^{\prime} \in X^{\prime} .
$$

Theorems 2 and 6 of (7) show that $T$ is decomposable on $J$ if and only if $T$ is well-bounded on $J$; and the two constants $K$ coincide. Also, we can choose $F(\cdot)$ so that $S^{\prime} F(s)=F(s) S^{\prime}(s \in R)$ for all $S$ in $L(X)$ satisfying $S T=T S$. Furthermore, the operational calculus of Lemma 9 is given by

$$
\left\langle f(T) x, x^{\prime}\right\rangle=f(b)\left\langle x, x^{\prime}\right\rangle-\int_{a}^{b}\left\langle x, F(s) x^{\prime}\right\rangle f^{\prime}(s) d s,
$$

where $x \in X, x^{\prime} \in X^{\prime}, f \in \mathscr{A}_{\mathrm{J}}$.

Let $T$ be a well-bounded operator on $X . T$ is said to be decomposable in $X$ if there is a family $\{\boldsymbol{E}(s): s \in \boldsymbol{R}\}$ of projections on $X$ such that $\left\{E^{\prime}(s): s \in \boldsymbol{R}\right\}$ is a decomposition of the identity for $T$. If an operator is decomposable in $X$, then it has a unique decomposition of the identity (7, Theorem 8 ).

Let $T$ be decomposable in $X$ with unique decomposition of the identity $\left\{E^{\prime}(s): s \in \boldsymbol{R}\right\}$. Following Berkson and Dowson (2), we say that $T$ is wellbounded of type (B) if $E \in \mathscr{E}_{J}$; that is, if $E(s+)=E(s)(s \in R)$ and $E(s-)$ is defined at each $s$ in $\boldsymbol{R}$.

The following theorem (cf. (2, Theorem 4.2)) characterises the well-bounded operators of type (B) in a manner similar to the characterisation of scalart ype spectral operators in (10). 
Theorem 5. Let $T$ be a bounded operator on the Banach space $X$. The following five conditions are equivalent:

(i) $T$ is well-bounded of type (B) with an $\mathscr{A}_{J}$-operational calculus of norm $K$;

(ii) there exist a compact interval $J$ and a naturally ordered family $\{E(s): s \in \boldsymbol{R}\}$ of projections on $X$ such that $E \in \mathscr{E}_{J}, E(b)=I, K=\sup _{\mathbb{R}}\|E(s)\|$ and

$$
T=\int_{J}^{\oplus} s d E(s)
$$

(iii) $T$ is well-bounded with an operational calculus $\psi: \mathscr{A}_{J} \rightarrow L(X)$ of norm $K$, such that $\psi\left(\left(\mathscr{A}_{J}\right)_{1}\right)$ is weakly relatively compact in $L(X)$;

(iv) $T$ is well-bounded with an operational calculus $\psi: \mathscr{A}_{J} \rightarrow L(X)$ of norm $K$, and $\psi_{x}$ is weakly compact for each $x$ in $X$;

(v) $T$ is well-bounded with an operational calculus $\psi: \mathscr{A}_{J} \rightarrow L(X)$ of norm $K$, and $\psi_{x}$ is compact for each $x$ in $X$.

Proof. We show that (i) $\Rightarrow($ v $) \Rightarrow$ (iv) $\Rightarrow$ (iii) $\Rightarrow($ ii) $\Rightarrow$ (i).

(i) $\Rightarrow$ (v). Let $\left\{E^{\prime}(s): s \in R\right\}$ be the unique decomposition of the identity for $T$. By the definition of a type (B) operator, $E \in \mathscr{E}_{J}$. We define $\psi: \mathscr{A}_{J} \rightarrow L(X)$ by

$$
\psi(f)=\int_{J}^{\oplus} f d E, f \in \mathscr{A}_{J} .
$$

The map $\psi$ is linear and bounded; also,

moreover,

$$
\psi(s \mapsto 1)=\int_{J}^{\oplus} d E=I ;
$$

$$
\begin{aligned}
\left\langle\psi(s \mapsto s) x, x^{\prime}\right\rangle & =\int_{J}^{\oplus} s d\left\langle E(s) x, x^{\prime}\right\rangle \\
& =b\left\langle x, x^{\prime}\right\rangle-\int_{J}\left\langle E(s) x, x^{\prime}\right\rangle d s \\
& =\left\langle T x, x^{\prime}\right\rangle, \quad x \in X, x^{\prime} \in X^{\prime} .
\end{aligned}
$$

Hence $\psi(s \mapsto s)=T$.

Since $E$ is naturally ordered,

$$
\begin{aligned}
\{f(a) E(a)+\Sigma f(E \Delta \bar{u})\}\{g(a) E(a)+\Sigma & g(E \Delta \bar{u})\} \\
& =f g(a) E(a)+\Sigma f g(E \Delta \bar{u}), \quad f, g \in \mathscr{A}_{J}, \quad \bar{u} \in \pi_{J} .
\end{aligned}
$$

Thus $\psi$ is an algebra homomorphism. By Lemma $9, \psi$ is the unique $\mathscr{A}_{J}$ operational calculus for $T$.

For each $x$ in $X$, the function $s \mapsto E(s) x$ is right continuous. Hence its range $\mathscr{E}_{x}=\{E(s) x: s \in R\}$ is separable, and its set of discontinuities is countable $(9,330)$.

Let $\left\{E\left(s_{n}\right) x: n \in N\right\}$ be a sequence of points in $\mathscr{E}_{x}$. Since $E(s)=0(s<a)$ and $E(s)=I(s \geqq b)$, we can assume that $s_{n} \in[a-\varepsilon, b]$ (any $\varepsilon>0$ ). We can 
therefore extract a monotone (convergent) subsequence $\left\{s_{n}^{\prime}\right\}$ from $\left\{s_{n}\right\}$. The sequence $\left\{E\left(s_{n}^{\prime}\right) x: n \in N\right\}$ converges because $E \in \mathscr{E}_{J}$ : thus $\mathscr{E}_{x}$ is relatively compact in $X$. Let $\mathscr{K}_{x}$ be the closed absolutely convex hull of $\mathscr{E}_{x}$. Then $\mathscr{K}_{x}$ is compact (by the argument of $(3, \mathrm{~V}, 2.6)$ ).

Let $f \in \mathscr{A}_{J},\|f\|_{J} \leqq 1$; let $\bar{u} \in \pi_{J}$. Then

$$
f(b) E(b) x-\Sigma E(f \Delta \bar{u}) x=f(b) x-\sum_{1}^{m}\left(f\left(u_{k}\right)-f\left(u_{k-1}\right)\right) E\left(u_{k}^{*}\right) x \in \mathscr{K}_{x} .
$$

Therefore $\psi_{x}(f) \in \mathscr{K}_{x}$. Thus $\psi_{x}$ is a compact map.

(v) $\Rightarrow$ (iv). Trivial.

(iv) $\Rightarrow$ (iii). This is immediate from Lemma 1 .

(iii) $\Rightarrow$ (ii). We define the function $k_{s, h}$ on $\boldsymbol{R}$ for each $s$ in $\boldsymbol{R}$ and $h>0$ by

$$
k_{s, h}(t)= \begin{cases}1, & t \leqq s, \\ 1+(s-t) / h, & s \leqq t \leqq s+h, \\ 0, & s+h \leqq t\end{cases}
$$

then $k_{s, h} \in \mathscr{A}_{J}$ and $\left\|k_{s, h}\right\|_{J} \leqq 1$. Also, $\chi_{(-\infty, s]}=\lim _{h \rightarrow 0} k_{s, h}$, pointwise.

Let $\mathscr{U}$ be an ultrafilter on $(0, \infty)$ converging to 0 in the usual topology of $\boldsymbol{R}$. When $f$ is a continuous function on $(0, \infty)$ we write $\lim f(h)$ for the value at $\mathscr{U}$ of the extension of $f$ to the Stone-Cech compactification of $(0, \infty)$.

Lemmas 2 and 4 of (7) show that every bounded functional on $\mathscr{A}_{J}$ has the form

$$
f \mapsto L(f)=m_{L} f(b)-\int_{a}^{b} \omega_{L}(s) f^{\prime}(s) d s, \quad f \in \mathscr{A}_{J},
$$

where $m_{L} \in C, \omega_{L} \in L^{\infty}(J)$ and

$$
\omega_{L}(s)=\lim _{\substack{h \rightarrow 0 \\ \mathscr{q}^{0}}} \int_{0}^{1} \omega_{L}(s+h t) d t, \quad a \leqq s<b .
$$

We define $L_{x, x^{\prime}}$ on $\mathscr{A}_{J}$ for $x$ in $X$ and $x^{\prime}$ in $X^{\prime}$ by

$$
L_{x, x^{\prime}}(f)=\left\langle\psi(f) x, x^{\prime}\right\rangle, \quad x \in X, x^{\prime} \in X^{\prime}, f \in \mathscr{A}_{J} .
$$

Let $m_{x, x^{\prime}}$ and $\omega_{x, x^{\prime}}$ be the associated constant and $L^{\infty}(J)$ function:

$$
L_{x, x^{\prime}}(f)=m_{x, x^{\prime}} f(b)-\int_{a}^{b} \omega_{x, x^{\prime}}(s) f^{\prime}(s) d s, \quad x \in X, x^{\prime} \in X^{\prime}, f \in \mathscr{A}_{J} .
$$

Then

$$
\left\langle\psi(s \mapsto 1) x, x^{\prime}\right\rangle=\left\langle x, x^{\prime}\right\rangle=m_{x, x^{\prime}}
$$

Also

The set

$$
L_{x, x^{\prime}}\left(k_{s, h}\right)=\int_{0}^{1} \omega_{x, x^{\prime}}(s+h t) d t, \quad a \leqq s<s+h<b .
$$

$$
\mathscr{K}=\left\{\psi\left(k_{s, h}\right): a \leqq s<s+h<b\right\}^{w}
$$


is compact and Hausdorff in the weak topology of $L(X)$ because $\psi\left(\left(\mathscr{A}_{J}\right)_{1}\right)$ is weakly relatively compact. $\mathscr{K}$ may therefore be considered as a complete Hausdorff uniform space with the uniformity defined by the functions

Since

$$
\left\{S \mapsto\left\langle S x, x^{\prime}\right\rangle: x \in X, x^{\prime} \in X^{\prime}\right\} \text {. }
$$

$$
\left\langle\psi\left(k_{s, h}\right) x, x^{\prime}\right\rangle=\int_{0}^{1} \omega_{x, x^{\prime}}(s+h t) d t \stackrel{h \rightarrow 0}{\rightarrow} \omega_{x, x^{\prime}}(s), \quad a \leqq s<b,
$$

the filter $\left\{\psi\left(k_{s, h}\right): h \in U, U \in \mathscr{U}\right\}$ is Cauchy, and therefore has a unique weak limit point, say $E(s)$, in $\mathscr{K}$. Let $E(s)=0(s<a)$ and $E(s)=I(s \geqq b)$.

Since $k_{s, h} k_{t, k}=k_{t, k} k_{s, h}=k_{s, h}$ for $0<h<t-s, 0<k$, we have

$$
E(s) E(t)=E(t) E(s)=E(s)
$$

when $s<t$. Thus $E: R \rightarrow L(X)$ is a naturally ordered function. By Lemma 3 and the weak compactness of $\mathscr{K}$, the strong limits $E(s+)$ and $E(s-)$ exist for all $s$ in $R$. Also, since

we have

$$
\left\langle E(s) x, x^{\prime}\right\rangle=\omega_{x, x^{\prime}}(s), \quad a \leqq s<b, x \in X, x^{\prime} \in X^{\prime},
$$

$$
\begin{aligned}
\left\langle E(s) x, x^{\prime}\right\rangle & =\lim _{\substack{h \rightarrow 0 \\
\mathscr{q}}} \int_{0}^{1} \omega_{x, x^{\prime}}(s+h t) d t \\
& =\omega_{x, x^{\prime}}(s+) \\
& =\left\langle E(s+) x, x^{\prime}\right\rangle, \quad a \leqq s<b .
\end{aligned}
$$

Therefore $E(s)=E(s+)(a \leqq s<b)$; hence each $E(s)$ is a projection. Thus $E \in \mathscr{E}_{J}$.

We define $\psi^{\prime}: \mathscr{A}_{J} \rightarrow L(X): f \mapsto \int_{J}^{\oplus} f d E$. Then

$$
\begin{aligned}
\left\langle\psi^{\prime}(f) x, x^{\prime}\right\rangle & =f(b)\left\langle x, x^{\prime}\right\rangle-\int_{J}\left\langle E(s) x, x^{\prime}\right\rangle d f(s) \\
& =f(b)\left\langle x, x^{\prime}\right\rangle-\int_{a}^{b} \omega_{x, x^{\prime}}(s) f^{\prime}(s) d s \\
& =\left\langle\psi(f) x, x^{\prime}\right\rangle, \quad x \in X, x^{\prime} \in X^{\prime}, f \in \mathscr{A}_{J} .
\end{aligned}
$$

So $\psi=\psi^{\prime}$ and $T=\int_{J}^{\oplus} s d E(s)($ take $f: s \mapsto s)$.

By Lemma 6, $\left\|\psi^{\prime}\right\| \leqq \sup _{R}\|E(s)\|$. Since $E(s)=\operatorname{st}_{h \rightarrow 0} \lim _{\substack{q \\ q_{s} h}} \psi\left(k_{s}\right)$ we see that $\left\|\psi^{\prime}\right\|=\sup _{\boldsymbol{R}}\|E(s)\|$.

(ii) $\Rightarrow$ (i). The operational calculus $\psi^{\prime}$ constructed above shows that (ii) $\Rightarrow$ (i).

The theorem is proved. 
We note that each projection $E(s)(a \leqq s<b)$ is the strong limit of the sequence $\left\{\psi\left(k_{s, n-s}\right): n \in N\right\}$.

Theorem 6. Let $T$ be a bounded operator on the Banach space $X$ satisfying the equivalent conditions of Theorem 5. Then the operational calculus $\psi$ extends to an operational calculus $\psi^{\prime}: \mathscr{B}_{J} \rightarrow L(X)$ having the same norm. Let $\left\{g_{\alpha}: \alpha \in \sigma\right\}$ be a uniformly bounded net in $\mathscr{B}_{\mathrm{J}}$ converging pointwise to a function $g$ in $\mathscr{B}_{J}$ : then $g(T)=\mathrm{st} \lim g_{\alpha}(T)$. Also $\left\{g(T): g \in \mathscr{B}_{J}\right\} \subset\left\{f(T): f \in \mathscr{A}_{J}\right\}^{s}$.

Proof. We define $\psi^{\prime}: \mathscr{B}_{J} \rightarrow L(X): f \mapsto \int_{J}^{\oplus} f d E$. It is clear that $\psi^{\prime}$ is a linear map of norm $\sup _{R}\|E(s)\|$, and that $\psi^{\prime}$ extends $\psi$. The argument in the proof of Theorem $5((\mathrm{i}) \Rightarrow(\mathrm{v}))$ shows that $\psi^{\prime}$ is an algebra homomorphism.

$\left\{g(T): g \in \mathscr{B}_{J}\right\} \subset\left\{f(T): f \in \mathscr{A}_{J}\right\}^{s}$ since $\{E(s): s \in R\} \subset\left\{f(T): f \in \mathscr{A}_{J}\right\}^{s}$ and the integrals defining $\psi^{\prime}$ exist in the strong topology.

The rest of the theorem follows directly from Theorem 2 .

\section{REFERENCES}

(1) J. Y. BARRY, On the convergence of ordered sets of projections, Proc. Amer. Math. Soc. 5 (1954), 313-314.

(2) E. Berkson and H. R. Dowson, On uniquely decomposable well-bounded operators, Proc. London Math. Soc. (3) 22 (1971), 339-358.

(3) N. Dunford and J. T. Schwartz, Linear operators (Interscience Publishers Inc., New York, 1958).

(4) E. HEwITT, A problem concerning finitely additive measures, Mat. Tidsskr. B 1951 (1951), 81-94.

(5) G. L. KRABBE, A Helly convergence theorem for Stieltjes integrals, Indagationes Math. 27 (1965), 52-69.

(6) J. R. RINGrose, On well-bounded operators, J. Australian Math. Soc. 1 (1960), 334-343.

(7) J. R. RINGrose, On well-bounded operators II, Proc. London Math. Soc. (3) 13 (1963), 613-638.

(8) W. H. Sills, On absolutely continuous functions and the well-bounded operator, Pacific J. Math. 17 (1966), 349-366.

(9) D. R. Smart, Conditionally convergent spectral expansions, J. Australian Math. Soc. 1 (1960), 319-333.

(10) P. G. SPaIn, On scalar-type spectral operators, Proc. Cambridge Phil. Soc. 69 (1971), 409-410.

\section{UNIVERSITY OF Glasgow}

GLASGOW, W.2 